\title{
Protective effect of parecoxib sodium against ischemia reperfusion-induced intestinal injury
}

\author{
MEI LI and ZHI ZHENG
}

\begin{abstract}
Department of Anesthesiology, Huangyan Hospital Affiliated to Wenzhou Medical University, Taizhou First People's Hospital, Taizhou, Zhejiang 318020, P.R. China
\end{abstract}

Received June 7, 2021; Accepted August 18, 2021

DOI: $10.3892 / \mathrm{mmr} .2021 .12416$

\begin{abstract}
Ischemia reperfusion (I/R)-induced intestinal injury is a pathophysiological process leading to oxidative stress and inflammatory responses, and revealing its underlying mechanisms is essential for developing therapeutic strategies. Cyclooxygenase (COX) has been reported to be involved in I/R injury. Parecoxib sodium, a selective inhibitor for COX-2, exerts protective effects, such as reducing I/R-induced injuries in the heart, kidney and brain. However, the potential role of parecoxib sodium in protecting the small intestine against I/R-induced injury has rarely been investigated. Therefore, the aim of the present study was to elucidate the effects and potential mechanisms of parecoxib sodium in I/R-induced intestinal injury. In total, 60 Sprague-Dawley rats were randomly divided into four groups: Control (sham operation) group, intestinal I/R group, $10 \mathrm{mg} / \mathrm{kg}$ parecoxib sodium-pre-treated $\mathrm{I} / \mathrm{R}(\mathrm{I} / \mathrm{R}+$ Pare/10) group and the $20 \mathrm{mg} / \mathrm{kg}$ parecoxib sodium-pre-treated I/R (I/R + Pare/20) group. A regular I/R model was established to induce the intestinal injury in rats. Parecoxib sodium at 10 or $20 \mathrm{mg} / \mathrm{kg}$ was intraperitoneally administered into rats in both $\mathrm{I} / \mathrm{R}+$ Pare groups once daily for 5 consecutive days prior to ischemia. Blood samples and small intestinal tissues were collected at $2 \mathrm{~h}$ after reperfusion. Changes in the levels of malondialdehyde, nitric oxide, interleukin (IL)-1 $\beta$, IL-8, intercellular cell adhesion molecule-1 and IL-10, as well as the total antioxidant capacity were determined using ELISA, as were the activities of superoxidase dismutase and myeloperoxidase. Furthermore, the protein expression levels of total caspase-3, cleaved caspase-3, Bcl-2 and Bax were examined via western blot analysis. In addition, the daily survival rate post-reperfusion was examined for 7 days. It was revealed that parecoxib sodium increased the
\end{abstract}

Correspondence to: Dr Zhi Zheng, Department of Anesthesiology, Huangyan Hospital Affiliated to Wenzhou Medical University, Taizhou First People's Hospital, 218 Hengjie Street, Taizhou, Zhejiang 318020, P.R. China

E-mail: zhengz_hyyy@sina.com

Key words: parecoxib sodium, ischemia reperfusion, intestinal injury, inflammation levels of antioxidants and suppressed the intestinal oxidative injury induced by I/R. Moreover, parecoxib sodium downregulated the expression levels of the proinflammatory factors, but upregulated the expression levels of anti-inflammatory factors. The results also demonstrated that parecoxib sodium attenuated I/R-induced apoptosis and increased the survival rate of rats. Thus, administration of parecoxib sodium prior to intestinal $\mathrm{I} / \mathrm{R}$ attenuated intestinal injury and increased the rat survival rate by inhibiting I/R-induced inflammation, oxidative stress and apoptosis.

\section{Introduction}

Ischemia causes an increase in intracellular and mitochondrial calcium levels by impairing ATPase-dependent ion transport, as well as reduces cellular ATP levels and intracellular $\mathrm{pH}$. The lack of ATP disrupts the mechanism regulating cell volume and induces the lysis of organelles and plasma membranes (1). Reperfusion promotes the production of reactive oxygen species (ROS), the sequestration of proinflammatory immunocytes in ischemic tissues, endoplasmic reticulum stress and the development of post-ischemic capillary no-reflow, which further enhance tissue injury. These events finally lead to the opening of mitochondrial permeability transition pores, which results in ischemia/reperfusion (I/R)-induced cell lysis and death (1).

Ischemia can cause myocardial infarction, stroke and peripheral vascular disease (1). At present, alteplase, a tissue plasminogen activator, is the only United States Food and Drug Administration (US FDA) approved clot-busting medication used to recanalize the thrombosed or occluded vasculature in ischemic stroke (2). Numerous factors can cause I/R, such as acute mesenteric ischemia, small intestine transplantation, intestinal obstruction, trauma or shock (3). The reperfusion following ischemia further aggravates damage and results in more severe intestinal injury compared with ischemia alone (4). The small intestine is highly sensitive to $I / R$ as mucosal intestinal injury often leads to bacterial translocation and initiates the inflammatory response (5). Thus, regulation of inflammatory responses and oxidative stress in the small intestine has significant clinical implications for patients with $I / R$ injury (6). I/R is usually accompanied by the extensive production of inflammatory cytokines, as well as with the activation of both the innate and adaptive immune 
responses (7), which further increases the permeability of blood vessels, the tissue aggregation of neutrophils (8), the production of proinflammatory cytokines and hypotension (5).

Cyclooxygenase (COX)-derived prostanoids, such as prostacyclin and prostaglandin $\mathrm{E}$, have been revealed to exert crucial roles in I/R-induced intestinal injuries $(9,10)$. Firstly, COX catalyzes arachidonic acid into prostaglandin endoperoxide (PGH2), then $\mathrm{PGH} 2$ is converted into prostaglandin (11). COX-1 and COX-2, two COX isoenzymes, have been identified and investigated (12). It has been revealed that COX-1 is involved in the synthesis of prostanoids and exists in the majority of cells under normal conditions. Conversely, COX-2 is often undetectable under normal conditions, but its expression is markedly increased under pathological conditions (13). For example, both COX-1 and COX-2 are involved in I/R-induced myocardial (14) and gastric injuries (15). Moreover, COX-2-deficient mice exhibit a significant reduction in damage after I/R insult (16). However, the underlying protective mechanisms remain unknown.

As a selective COX-2 inhibitor, parecoxib sodium has exhibited potent capacities, such as relieving perioperative pain and reducing I/R-induced hepatic (17), cerebral (18) or renal injuries (19). Thus, the present study aimed to reveal the protective effect of parecoxib sodium in I/R-induced intestinal injury, particularly on the inflammatory response and oxidative stress. Moreover, the study aimed to further the understanding of the role of COX-2 in intestinal I/R injury.

\section{Materials and methods}

Animals. A total of 60 adult male Sprague-Dawley rats (age, 8-10 weeks; weight, 250-280 g) were obtained from the Animal Centre of Wenzhou Medical University (Wenzhou, China). Rats were individually housed in standard cages in rooms with a constant $\sim 21-25^{\circ} \mathrm{C}$ temperature and $\sim 50-65 \%$ relative humidity and a $12 \mathrm{~h}$-light/dark cycle. Rats were fed with standard chow and had free access to water. All animals received humane care according to the Guide for the Care and Use of Laboratory Animals of the National Institutes of Health. All animal care and experimental procedures were approved by the Wenzhou Medical University Animal Policy and Welfare Committee (approval no. 2013/APWC/0361). At the end of the experimental period, animals were euthanized via $\mathrm{CO}_{2}$ asphyxiation with a flow rate of $30 \%$ chamber vol $/ \mathrm{min}$.

Drug treatment. In total, 60 rats were randomly divided into four groups with 15 rats in each group: Control (sham operation) group, intestinal I/R group, $10 \mathrm{mg} / \mathrm{kg}$ parecoxib sodium-pre-treated I/R (I/R + Pare/10) group and $20 \mathrm{mg} / \mathrm{kg}$ parecoxib sodium-pre-treated I/R (I/R + Pare/20) group. Parecoxib sodium (Pfizer, Inc.) was diluted in isotonic saline. The prepared parecoxib sodium at 10 or $20 \mathrm{mg} / \mathrm{kg}$ was intraperitoneally injected into rats once daily for 5 consecutive days prior to ischemia. The parecoxib dosage was determined based on a previous study (19). Rats in sham and I/R groups were injected intraperitoneally with the same volume of isotonic saline at the same time. On the day of surgery, $1 \mathrm{~h}$ after the last injection of parecoxib sodium or saline, all animals were anesthetized with intraperitoneal injection of pentobarbital at $40 \mathrm{mg} / \mathrm{kg}$. The superior mesenteric artery was isolated and then completely occluded to induce ischemia. After $1 \mathrm{~h}$ of ischemia, reperfusion was initiated by removing the occlusion and was maintained for another $2 \mathrm{~h}$. Rats were covered with warm blankets to maintain their body temperature. At the end of reperfusion, blood and small intestinal tissue samples were harvested and used for subsequent assays. The time period for ischemia and reperfusion was determined based on a previous study (20). The survival rate was calculated daily for 7 consecutive days after reperfusion. Once rats reach the criteria of humane endpoints [a reduction of $\sim 4-6^{\circ} \mathrm{C}$ in body temperature, a weight loss of $>10 \%$, decreased activity (lethargy) and alertness, a rough coat and hunched posture, which are direct signs of illness, pain or distress], they were euthanized immediately. While rats that survived for 7 days received euthanasia right after experiments via $\mathrm{CO}_{2}$ asphyxiation with a flow rate of $30 \%$ chamber $\mathrm{vol} / \mathrm{min}(21)$.

Biochemical assays. The total antioxidant capacity (TAC) and malondialdehyde (MDA) levels in the serum and small intestinal samples were evaluated using a TAC Assay kit (cat. no. S0119; Beyotime Institute of Biotechnology) and a MDA assay kit (cat. no. S0131S; Beyotime Institute of Biotechnology), respectively. Tissues stored at $-80^{\circ} \mathrm{C}$ were homogenized in cold PBS at $2-8^{\circ} \mathrm{C}$. Each homogenized sample was centrifuged at $4,000 \mathrm{x}$ g for $15 \mathrm{~min}$ at $4^{\circ} \mathrm{C}$, then the supernatant was collected for the detection of nitric oxide (NO) levels using the nitrate reductase method.

The activity of superoxidase dismutase (SOD) in the serum and small intestinal samples was assessed using the xanthine oxidase method, with the Total Superoxide Dismutase Assay kit supplied by Beyotime Institute of Biotechnology (cat. no. S0109). SOD activity was measured using a microplate reader at a wavelength of $560 \mathrm{~nm}$. Myeloperoxidase (MPO) activity was measured using the spectrophotometric method with 3,3-5,5 tetramethylbenzine as the substrate, and the light absorbance was measured at $460 \mathrm{~nm}$ over a period of $5 \mathrm{~min}$ (Elabscience Biotechnology, Inc.; cat. no. E-BC-K074-S). The values of SOD and MPO activity were presented as unit per $\mu \mathrm{l} / 1$ of serum or unit per $\mu \mathrm{g} / \mathrm{g}$ of tissue. All detection procedures were performed in accordance with the manufacturer's instructions.

ELISA. ELISA kits for interleukin (IL)-1 $\beta$ (cat. no. RLB00; R\&D Systems, Inc.),IL-8 (cat.no. RA20553; Bioswamp; Wuhan Bienle Biotechnology Co., Ltd.), intercellular cell adhesion molecule-1 (ICAM-1; cat. no. RIC100; R\&D Systems, Inc.) and IL-10 (cat. no. R1000; R\&D Systems, Inc.) were commercially purchased and used to determine the concentration of each cytokine both in the plasma and small intestinal samples. In total, a 100- $\mu 1$ aliquot of the supernatant from each well was collected for measurement. The concentration of each cytokine in the supernatant was standardized to the cell protein concentration in the respective well. The absorbance was measured at $450 \mathrm{~nm}$ using a Model 550 microplate reader (Bio-Rad Laboratories, Inc.). The experiments were repeated four times using different batches of rats.

Western blot analysis. Frozen small intestinal samples were homogenized in RIPA buffer freshly supplemented with protease and phosphatase inhibitor cocktails (Sigma-Aldrich; 
Table I. Summary of primary antibodies used in the present study.

\begin{tabular}{|c|c|c|c|c|}
\hline Antibody & cat. no. & Host & Vendor & Dilution \\
\hline Caspase-3 & sc-56053 & Mouse & Santa Cruz Biotechnology, Inc. & $1: 200$ \\
\hline Bcl-2 & ab194583 & Rabbit & Abcam & $1: 500$ \\
\hline Bax & ab182733 & Rabbit & Abcam & $1: 500$ \\
\hline$\beta$-actin & sc-47778 & Mouse & Santa Cruz Biotechnology, Inc. & $1: 200$ \\
\hline
\end{tabular}

MerckKGaA) on ice.Lysates wereharvested aftercentrifugation at $12,000 \times \mathrm{g}$ for $30 \mathrm{~min}$ at $4^{\circ} \mathrm{C}$. The protein concentration was determined with a Bradford assay. Then, $30 \mu \mathrm{g}$ protein for each sample was loaded and run via 10\% SDS-PAGE, following which it was transferred onto a PVDF membrane (Roche Diagnostics). After blocking with 5\% fat-free milk at room temperature for $1 \mathrm{~h}$, membranes were incubated with specific primary antibodies at their corresponding dilution (Table I) at $4^{\circ} \mathrm{C}$ overnight. The following day, membranes were rinsed and incubated with HRP-conjugated secondary antibodies at 1:1,000 dilution (bovine anti-rabbit IgG-HRP, cat. no. sc-2370; bovine anti-mouse IgG-HRP, cat. no. sc-2371; Santa Cruz Biotechnology, Inc.) at room temperature for $1 \mathrm{~h}$. The protein bands were developed using ECL Advance Detection kits (Thermo Fisher Scientific, Inc.) and detected with a Multi Image Light Cabinet (Protein Simple). Densitometric analysis of each band was performed using ImageJ software (version 1.46; National Institutes of Health).

Statistical analysis. Statistical analysis was conducted by using GraphPad Prism 5.0 software (GraphPad Software, Inc.). All results were presented as the mean \pm standard deviation (SD) of at least three independent experiments. All data were compared by one-way ANOVA that was followed by Tukey's post hoc test. Survival rates were analyzed by the Kaplan-Meier method using a log-rank test. $\mathrm{P}<0.05$ was considered to indicate a statistically significant difference.

\section{Results}

Parecoxib sodium pre-administration attenuates IR-induced oxidative stress both in the serum and small intestinal tissues. In order to evaluate the protective effect of parecoxib sodium against oxidative stress, several parameters in the serum were detected, including the TAC, the concentrations of MDA and NO, and the activities of SOD and MPO. As revealed in Fig. 1A, I/R significantly increased the serum concentration of MDA, which is a product of lipid peroxidation, by $\sim 2$-fold, while the administration of parecoxib sodium significantly inhibited I/R-induced MDA levels by $30-50 \%$. The serum concentration of NO was increased by $\sim 3.5$-fold post-I/R, while parecoxib sodium decreased NO levels by $50-70 \%$ (Fig. 1B). Conversely, I/R significantly decreased TAC in the serum by $60 \%$, while parecoxib sodium significantly increased TAC by $1.8-2$-fold (Fig. 1C). Moreover, I/R significantly suppressed the serum SOD activity by $75 \%$ and enhanced MPO activity by 3.5 -fold, while parecoxib sodium pre-treatment restored SOD activity close to the control level and inhibited I/R-induced MPO activation in a dose-dependent manner (Fig. 1D and E).
Next, the protective effect of parecoxib sodium against oxidative stress in small intestinal tissues was examined. As revealed in Fig. 2A and B, parecoxib sodium re-treatment significantly suppressed I/R-induced MDA and NO intestinal levels, and restored them close to the control levels. Moreover, parecoxib sodium significantly enhanced TAC intestinal levels and restored SOD activity (Fig. 2C and D). It was also revealed that parecoxib sodium inhibited I/R-induced MPO activity to a level that was close to that of the control (Fig. 2E). These results from intestinal tissues were consistent with corresponding results from serum samples revealed in Fig. 1.

Parecoxib sodium pre-administration decreases the inflammatory responses both in the serum and small intestinal tissues. To evaluate the inhibitory effect of parecoxib sodium on the inflammatory responses, the serum levels of proinflammatory cytokines, including IL-1 $\beta$ (Fig. 3A), IL-8 (Fig. 3B) and ICAM-1 (Fig. 3C), as well as the serum level of the anti-inflammatory cytokine IL-10 (Fig. 3D), were assessed. ELISA results demonstrated that the serum levels of IL-1 $\beta$, IL-8 and ICAM-1 were significantly increased by 4-5-fold after I/R injury, while pre-treatment with parecoxib sodium could significantly suppress IL-1 $\beta$, IL- 8 and ICAM- 1 levels by 50-75\% (Fig. 3A-C). Conversely, I/R decreased IL-10 serum levels by $70 \%$, while parecoxib sodium exerted an inhibitory effect on I/R-induced IL-10 downregulation (Fig. 3D).

Next, the levels of IL-1 $\beta$ (Fig. 4A), IL-8 (Fig. 4B), ICAM-1 (Fig. 4C) and IL-10 (Fig. 4D) were assessed in small intestinal tissues post-I/R or I/R + Pare treatment. The results demonstrated that parecoxib sodium significantly decreased I/R-induced IL-1 $\beta$, IL- 8 and ICAM-1 levels by $60-75 \%$ (Fig. 4A-C). In addition, parecoxib sodium significantly enhanced IL-10 levels in the small intestine, which had been reduced by I/R (Fig. 4D).

Parecoxib sodium pre-administration reduces $I / R$-induced apoptosis. To evaluate the inhibitory effect of parecoxib sodium on apoptosis, small intestinal tissues subjected to I/R or $\mathrm{I} / \mathrm{R}+$ Pare treatment were collected and the protein expression levels of several apoptosis-related markers were examined, including the total caspase-3 (Fig. 5A), cleaved caspase-3 (Fig.5A), Bcl-2 (Fig. 5E) and Bax (Fig. 5E). Western blot analysis revealed that, although both $\mathrm{I} / \mathrm{R}$ and $\mathrm{I} / \mathrm{R}+$ Pare did not induce an obvious change on total caspase-3 expression (Fig. 5B), I/R significantly induced the production of the cleaved caspase- 3 (Fig. 5C) and Bax (Fig. 5G) by 5-fold and 3-fold, respectively. However, pre-treatment of parecoxib sodium significantly inhibited I/R-induced cleaved caspase-3 activation (Fig. 5C) and Bax expression (Fig. 5G). Conversely, parecoxib sodium 
A

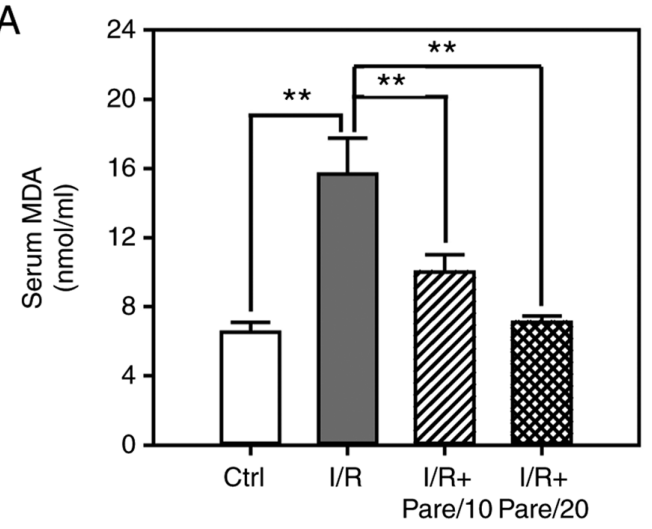

C

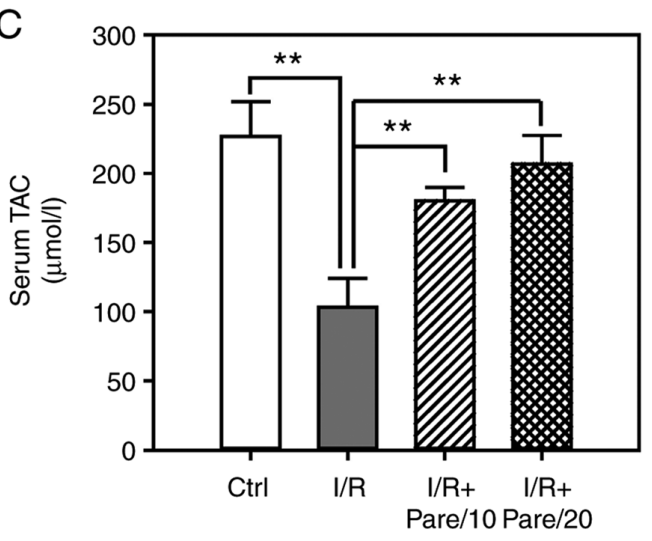

B

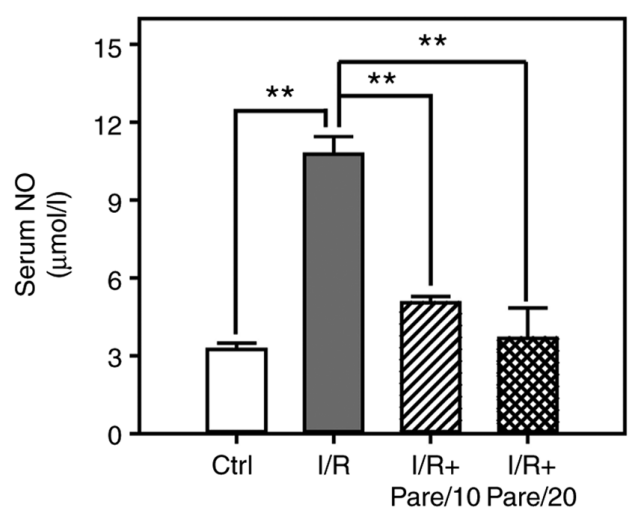

D

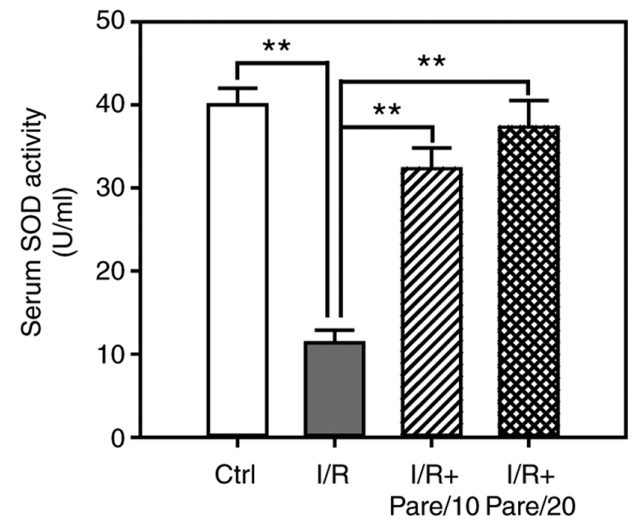

E

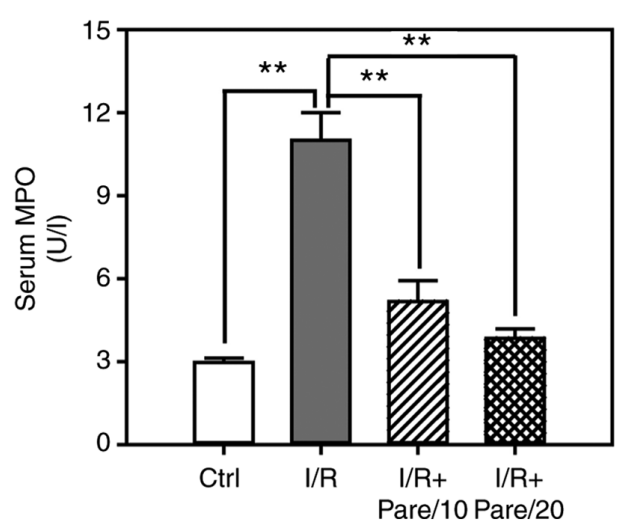

Figure 1. Assessment of the oxidative stress after $\mathrm{I} / \mathrm{R}$ with/without parecoxib sodium pre-treatment in rat serum. (A-C) The contents of malondialdehyde (A) nitric oxide (B) and total antioxidant capacity (C) in rat serum in control, I/R, I/R + Pare/10, and I/R + Pare/20 groups. (D and E) The activities of superoxidase dismutase (D) and myeloperoxidase (E) in rat serum in each group. Bars represent the means $\pm \mathrm{SD}, \mathrm{n}=3$. ${ }^{* *} \mathrm{P}<0.01 \mathrm{vs}$. the $\mathrm{I} / \mathrm{R}$ group. $\mathrm{I} / \mathrm{R}$, ischemia reperfusion; Pare, parecoxib; MDA, malondialdehyde, NO, nitric oxide; TAC, total antioxidant capacity; SOD, superoxidase dismutase; MPO, myeloperoxidase; Ctrl, control.

could restore I/R-induced Bcl-2 downregulation (Fig. 5F). It is worth noting that the ratio of cleaved caspase-3/total caspase-3, which indicates the activation of caspase-3, was significantly decreased in the parecoxib sodium-pre-treated group after I/R (Fig. 5D). Furthermore, the ratio of Bcl-2/Bax was recovered in the parecoxib sodium-pre-treated group after I/R (Fig. 5H). These results indicated the enhanced apoptosis after I/R, and that parecoxib sodium could efficiently suppress this effect.

Parecoxib sodium pre-treatment improves the survival rate of rats after I/R. Finally, it was examined whether parecoxib sodium exerted a protective role on the survival of rats after
$\mathrm{I} / \mathrm{R}$ treatment. The results demonstrated that $\sim 20 \% \mathrm{I} / \mathrm{R}$ model rats survived 7 days after intestinal $\mathrm{I} / \mathrm{R}$, while parecoxib sodium significantly improved the survival rate to $>65 \%$ after I/R (Fig. 6).

\section{Discussion}

The present study systematically examined the antioxidant activity of parecoxib sodium and investigated its role in I/R-induced small intestinal injuries. The results indicated that parecoxib sodium was a potent free radical scavenger, as well as a lipid peroxidation inhibitor. The pre-administration 
A

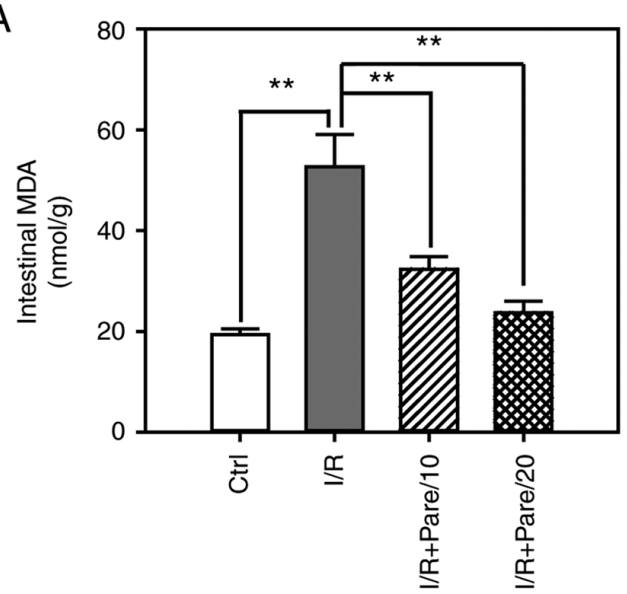

C

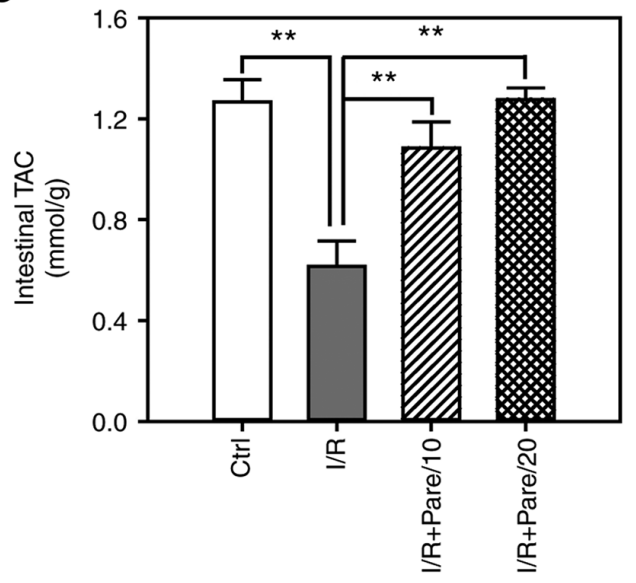

B

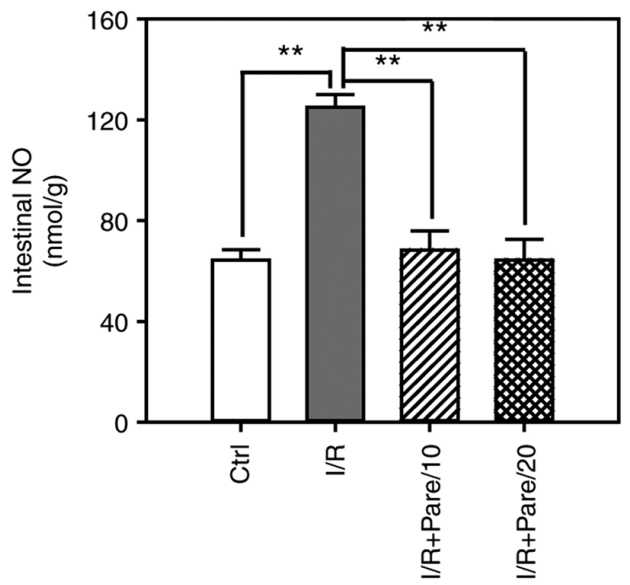

D

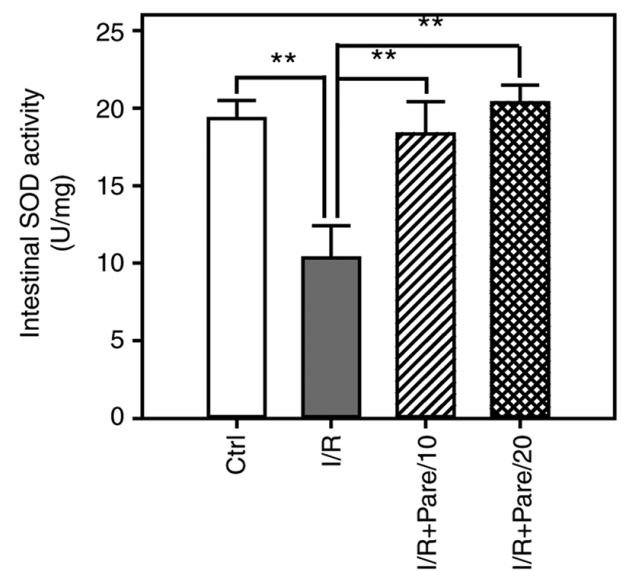

E

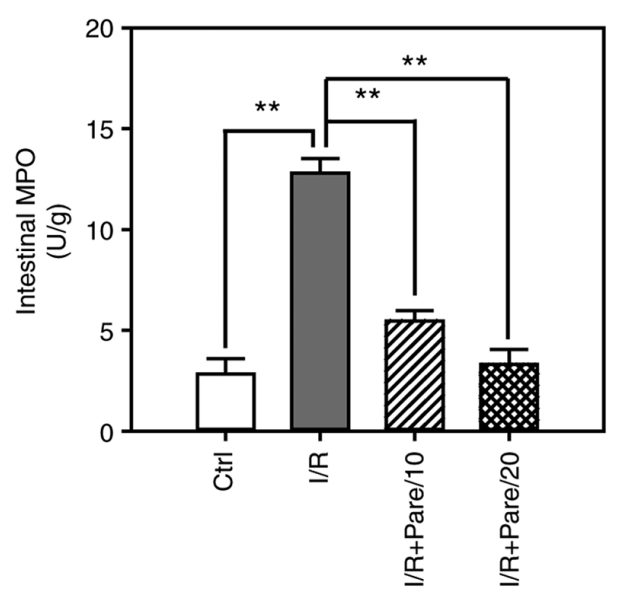

Figure 2. Assessment of the oxidative stress after I/R with/without parecoxib sodium pre-treatment in rat small intestinal tissues. (A-C) The contents of malondialdehyde (A) nitric oxide (B) and total antioxidant capacity (C) in the small intestine of rats in control, I/R, I/R + Pare/10 and I/R + Pare/20 groups. (D and E) The activities of superoxidase dismutase (D) and malondialdehyde (E) in rat small intestine in each group. Bars represent the means \pm SD, $n=3$. ${ }^{* *} \mathrm{P}<0.01$ vs. the I/R group. I/R, ischemia reperfusion; Pare, parecoxib; MDA, malondialdehyde, NO, nitric oxide; TAC, total antioxidant capacity; SOD, superoxidase dismutase; MPO, myeloperoxidase; Ctrl, control.

of parecoxib sodium significantly ameliorated numerous antioxidative biomarkers (such as MDA, NO, TAC, SOD and MPO), attenuated the inflammatory responses (IL-1 $\beta$, IL-6, ICAM-1 and IL-10), suppressed intestinal apoptosis (caspase-3, Bcl-2 and Bax) and improved the survival rate in rats after $\mathrm{I} / \mathrm{R}$ injury. Thus, the administration of parecoxib sodium prior to $\mathrm{I} / \mathrm{R}$ appears to protect rats against intestinal I/R injury.
$\mathrm{I} / \mathrm{R}$ is a common pathophysiological process and is accompanied with changes in the expression of COX in multiple organs, such as the small intestine $(10,22)$. COX-1 and COX-2 have both been revealed to contribute to I/R-induced intestinal injury. In the present study, the protective role of parecoxib sodium, a widely used and selective COX-2 inhibitor during the perioperative period, 
A



C

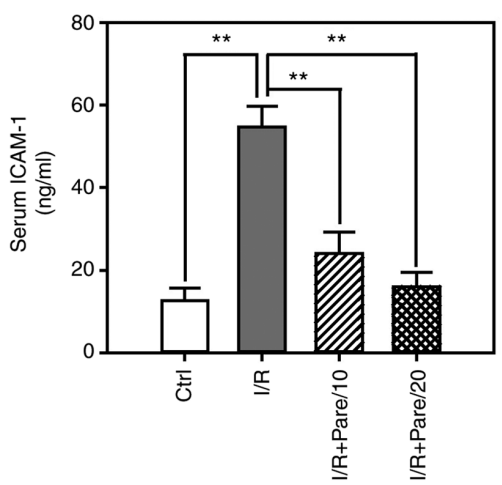

B

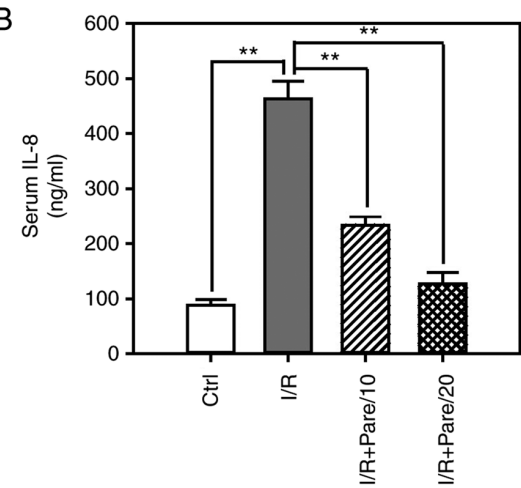

D

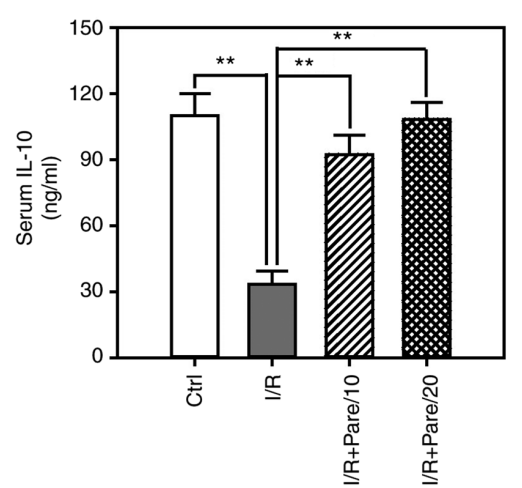

Figure 3. Assessment of the inflammatory response after I/R with/without parecoxib sodium pre-treatment in rat serum. (A-D) The concentrations of IL-1 $\beta$ (A) IL-8 (B) intercellular cell adhesion molecule-1 (C) and IL-10 (D) in rat serum in control, I/R, I/R + Pare/10, and I/R + Pare/20 groups were examined by ELISA. Bars represent the means $\pm \mathrm{SD}, \mathrm{n}=3$. ${ }^{* *} \mathrm{P}<0.01$ vs. the $\mathrm{I} / \mathrm{R}$ group. I/R, ischemia reperfusion; Pare, parecoxib; ICAM-1, intercellular cell adhesion molecule-1; IL, interleukin; Ctrl, control.

A

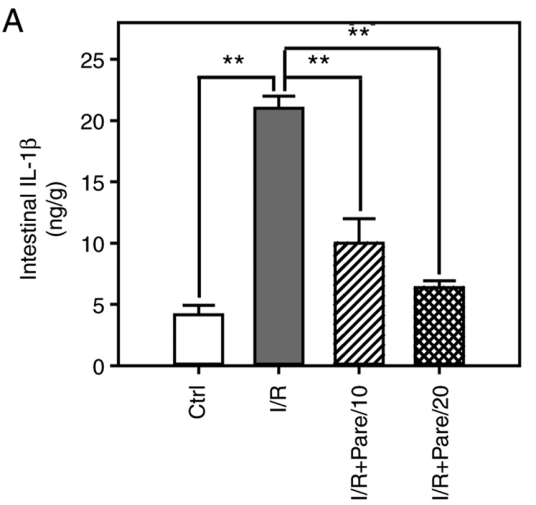

C

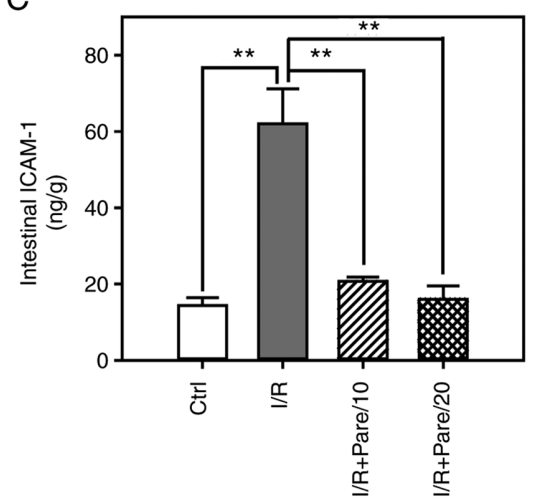

B

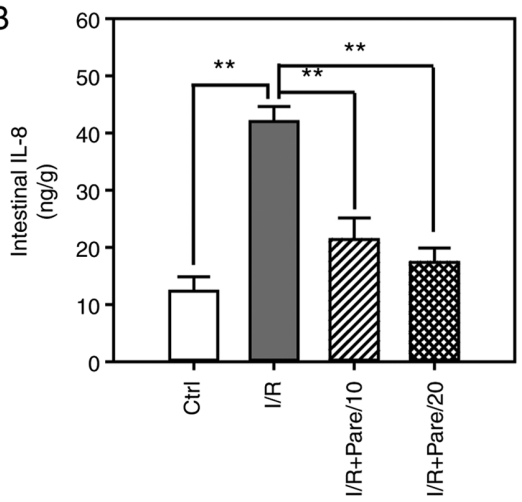

D



Figure 4. Assessment of the inflammatory response after I/R with/without parecoxib sodium pre-treatment in rat small intestinal tissues. (A-D) The concentrations of IL-1ß (A) IL-8 (B) intercellular cell adhesion molecule-1 (C) and IL-10 (D) in the small intestine of rats in each group were examined by ELISA. Bars represent the means $\pm \mathrm{SD}, \mathrm{n}=3 .{ }^{* *} \mathrm{P}<0.01$ vs. the I/R group. I/R, ischemia reperfusion; IL, interleukin; Pare, parecoxib; ICAM-1, intercellular cell adhesion molecule-1; Ctrl, control. 
A

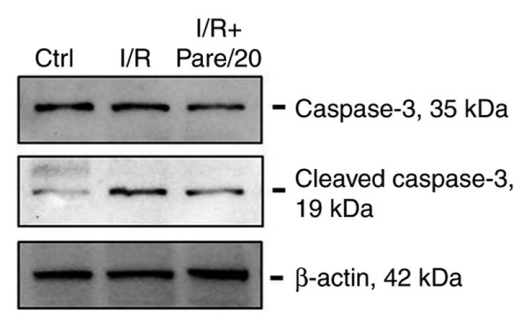

C

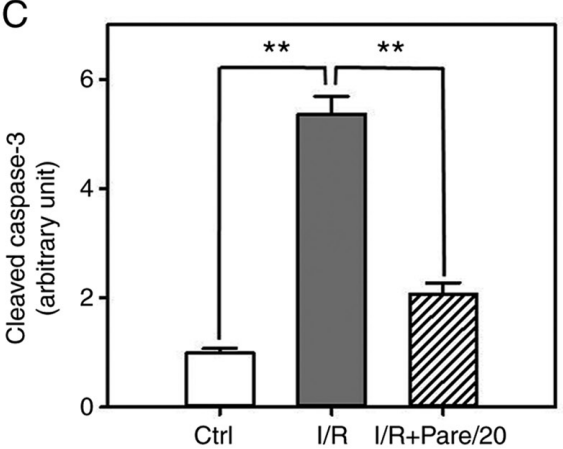

E
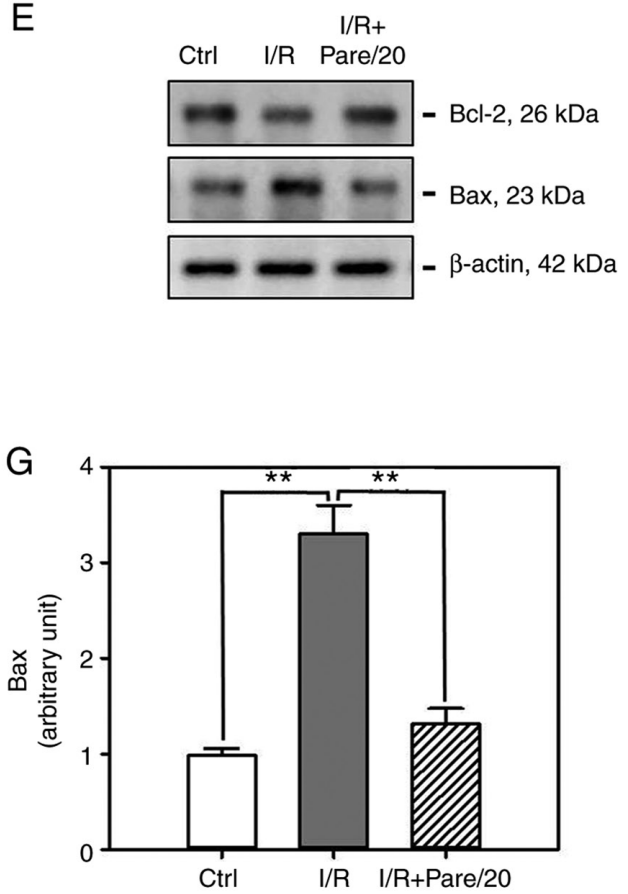

B

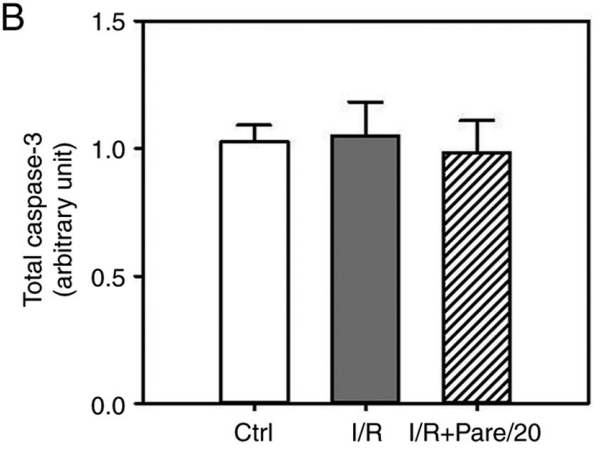

D



F



$\mathrm{H}$

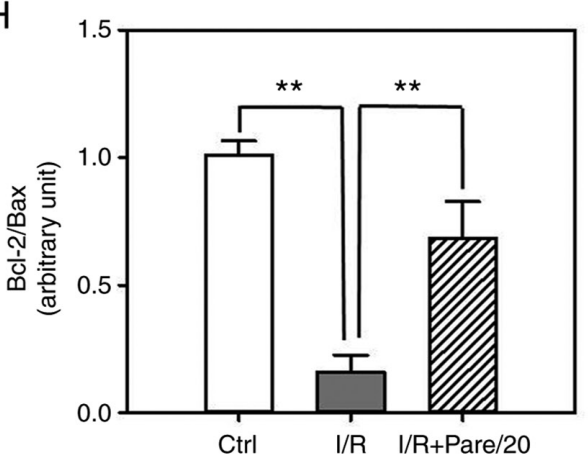

Figure 5. Assessment of the apoptosis-related protein expression after I/R with/without parecoxib sodium pre-treatment in small intestinal tissues. (A) The protein levels of total caspase- 3 and cleaved caspase- 3 in the small intestine of rats in control, I/R and I/R + Pare/20 groups were assessed by western blotting. (B-D) Densitometric analysis of immunoblotting data in panel A. (E) The protein levels of Bcl-2 and Bax in rat small intestine in control, I/R and I/R + Pare/20 groups were assessed by western blotting. (F-H) Densitometric analysis of immunoblotting data in panel E. Each bar is the mean \pm SD of three experiments, ${ }^{* * *} \mathrm{P}<0.01$ vs. the I/R group. I/R, ischemia reperfusion; Pare, parecoxib; Ctrl, control.

in small intestinal $\mathrm{I} / \mathrm{R}$ injury was evaluated, and the results demonstrated the essential role of COX in intestinal I/R injury.

Inflammatory cascades exert a critical role during I/R-induced tissue damage, and COX-2 is an important inflammatory mediator. Kamel et al (23) reported that modafinil significantly downregulated I/R-induced COX-2 upregulation and attenuated the elevated intestinal TNF- $\alpha$ and IL-1 $\beta$ levels. Moreover, Duarte et al (24) observed that myeloid COX-2 deletion led to a transient increase in IL-6 levels after hepatic I/R, while administration of celecoxib, a selective COX-2 inhibitor, significantly improved liver function in 


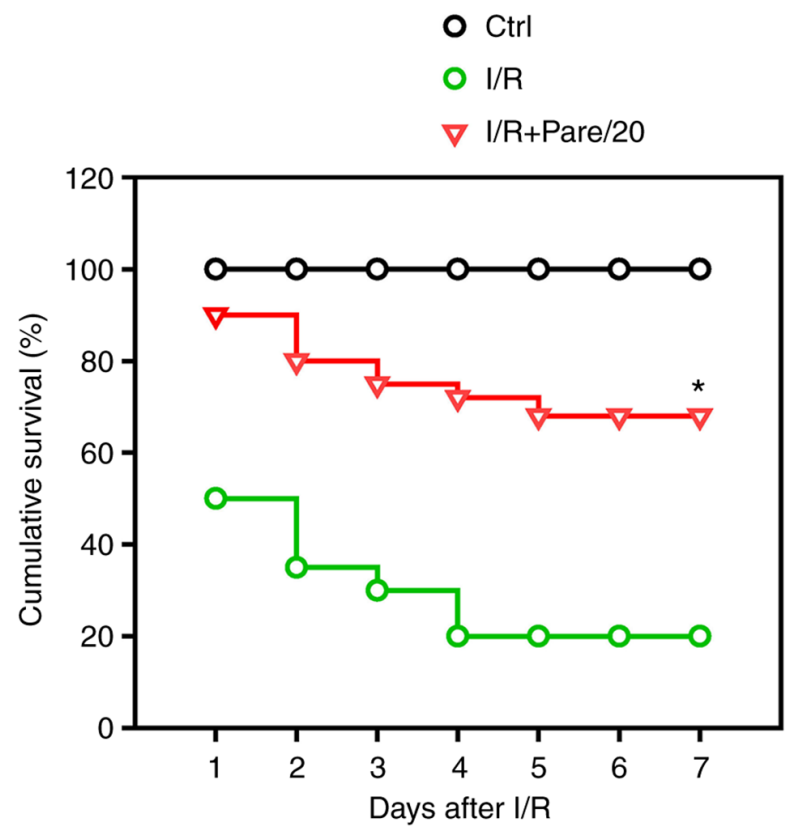

Figure 6. Effect of pre-administration of parecoxib sodium on the survival rate after intestinal I/R in rats. Survival rates over a 7-day period following intestinal I/R were recorded and calculated by Kaplan-Meier method using a log-rank test. " $\mathrm{P}<0.05$ vs. the I/R group. I/R, ischemia reperfusion; Pare, parecoxib; Ctrl, control.

$\mathrm{COX}-2^{-/-}$mice. Feng et al (25) also revealed that pre-operative administration of rofecoxib, another selective COX-2 inhibitor, decreased the serum levels of TNF- $\alpha$ and IL- 6 in patients, as well as inhibited both systemic and local inflammation. IL-8 is one of the major inflammatory mediators in intestinal $\mathrm{I} / \mathrm{R}$ injury and it exerts deleterious effects on the intestinal mucosa $(26,27)$. A recent study revealed that when COX-2 expression was suppressed by Ginkgo biloba, IL-8 levels were also reduced (28).

Accumulating evidence has shown that inflammatory reactions exert a critical function in I/R-induced intestinal damage, and inhibition of the inflammatory response efficiently protects intestinal tissues against injury (29). Parecoxib sodium, as a COX-2 inhibitor, exerts a potent anti-inflammatory effect (30). In the present study, it was revealed that parecoxib sodium pre-treatment decreased both the serum and tissue contents of several pro-inflammatory cytokines, such as IL-1 $\beta$, IL-8 and ICAM-1, while it increased the level of the anti-inflammatory cytokine IL-10. IL-1 $\beta$ and IL- 8 are mainly secreted by the activated M1-macrophages that possess various biological activities, and they act as important inflammatory mediators during I/R-induced intestinal injury (31). In I/R-induced tissue injury, both the levels of IL-1 $\beta$ and IL- 8 are increased at the early stage, accompanied with the increased permeability of intestinal epithelia, neutrophil adhesion and infiltration, and secretion of other cytokines, which further deteriorate the injured tissue $(23,27)$. In the present study, the enhanced ICAM-1 levels in the serum and small intestinal tissues after intestinal I/R may cause increased neutrophil infiltration, while inhibition of ICAM-1 by parecoxib sodium may ameliorate tissue injury. This finding was consistent with a previous study that reported leukocyte activation, neutrophil adhesion to endothelial cells and ultimately infiltration into I/R-injured tissues (32). Thus, these results indicated that ICAM-1 may serve as a candidate for therapeutic targeting. Collectively, the aforementioned findings demonstrated that parecoxib sodium could potently suppress the inflammatory response induced by I/R injury, which maybe characteristic of its preventive role in the small intestine.

In addition, recent studies have confirmed the role of parecoxib sodium in other diseases. For example, parecoxib sodium was found to inhibit the inflammatory response by inhibiting COX-2 expression and exhibited protective effects against sepsis in mice (33). Administration of parecoxib prior to hepatic I/R was revealed to attenuate hepatic injury via the inhibition of the inflammatory response and nitrosative stress (17). Furthermore, modafinil, a US FDA-approved novel wake-promoting agent, was revealed to exert a protective effect in intestinal I/R-induced injury in rats (20).

MDA is the final product resulting from lipid breakdown and oxidative stress, and it is considered as a good indicator of free radical-induced lipid peroxidation (34). In the present study, the contents of MDA were significantly increased both in the serum and small intestinal tissues from rats after I/R induction, while parecoxib sodium pre-administration significantly suppressed MDA levels in a dose-dependent manner. This result indicated that parecoxib sodium may suppress I/R-induced lipid peroxidation, enhance the antioxidant defense system, and thus, alleviate the oxidative injuries in small intestinal tissues.

NO acts as a double-edged sword in organisms. Under normal physiological conditions, NO is a helpful messenger and modulator. However, under oxidative stress, NO may be a toxic metabolite and can be harmful for intestinal mucosa. For example, a high level of exogenous NO was revealed to increase the degree of intestinal mucosal injury (35). Peroxynitrite, an oxidation product from the reaction of NO with superoxide, can promote the apoptosis of intestinal epithelial cells via the activation of caspases and the release of apoptosis-activating factor-1 in mitochondria (36). In the present study, NO levels were significantly enhanced, cleaved caspase- 3 was markedly activated and the ratio of Bcl-2/Bax was suppressed by I/R, which was consistent with a previous study (37).

The present findings are important to clarifying the antioxidant mechanism of parecoxib sodium in detail. There is an intricate balance between the production of ROS and its consumption in living organisms, and excessively released ROS will react with multiple macromolecules, including DNA, lipids and proteins (38). The body has developed an antioxidant defense system against the harmful effects of ROS. SOD is one of most critical enzymes in the cellular antioxidant system that can remove excessive ROS in living organisms (39). Therefore, modulating the contents of SOD in the serum or in the tissues may be able to protect against oxidative stresses. The present results demonstrated that I/R significantly inhibited SOD activity both in the serum and small intestinal tissues, while parecoxib sodium significantly restored the enzymatic activity of SOD in a dose-dependent manner, which may be responsible for the increased resistance to oxidative stress. Consistent with a recent study by Wu et al (40), parecoxib sodium reduced the levels of ROS and lipid peroxidation in myocardial I/R injury rats, thereby reducing oxidative stress. Similar findings were also observed in the TAC both in the serum and tissues. 
Along with the anti-inflammatory and anti-oxidative features of parecoxib sodium, the present study also evaluated whether parecoxib sodium was capable of improving I/R-induced intestinal apoptosis. Caspase-3, as one of the major signaling pathways involved in apoptosis, is a critical executing factor during apoptosis (41). The current results identified a significant activation of cleaved caspase-3 post-I/R, which was in agreement with a previous study reporting that activation of caspase- 3 was usually increased in intestinal I/R (41). However, the pre-treatment of parecoxib sodium effectively decreased cleaved caspase-3 expression, and thus, inhibited intestinal apoptosis.

In conclusion, to the best of our knowledge, the present study was the first to systematically examine the antioxidant activity of parecoxib sodium using an in vivo I/R rat model and to investigate the protective role against I/R-induced intestinal injury. The results demonstrated that parecoxib sodium decreased the inflammatory response, attenuated oxidative stress, suppressed apoptosis, and thus, increased the survival rate of rats. These results indicated a potential protective effect of parecoxib sodium against oxidative tissue damage, and that it could further improve the antioxidant defense system. Therefore, parecoxib sodium pre-treatment may be an effective strategy against I/R-induced intestinal injury in patients with intestinal obstruction, acute mesenteric ischemia, trauma, shock, neonatal necrotizing enterocolitis or small intestine transplantation due to its anti-inflammatory, anti-oxidative and anti-apoptotic properties. However, studies on parecoxib sodium-involved signaling mechanism, the upstream and downstream regulating molecules, cellular homeostasis and other biological functions are urgently required. Moreover, whether the molecular findings obtained from animal studies can be applied to clinical practice needs further exploration.

\section{Acknowledgements}

Not applicable.

\section{Funding}

No funding was received.

\section{Availability of data and materials}

The datasets used and/or analyzed during the current study are available from the corresponding author on reasonable request.

\section{Authors' contributions}

ML and $\mathrm{ZZ}$ conducted the experiments, analyzed the data, and wrote and revised the manuscript. All authors read and approved the final submission. ML and $\mathrm{ZZ}$ confirm the authenticity of all the raw data.

\section{Ethics approval and consent to participate}

All animal care and experimental procedures were approved (approval no. 2013/APWC/0361) by the Wenzhou Medical University Animal Policy and Welfare Committee (Wenzhou, China).

\section{Patient consent for publication}

Not applicable.

\section{Competing interests}

The authors declare that they have no competing interests.

\section{References}

1. Kalogeris T, Baines CP, Krenz $M$ and Korthuis RJ: Ischemia/reperfusion. Compr Physiol 7: 113-170, 2016.

2. Mandalaneni K, Rayi A and Jillella DV: Stroke reperfusion injury. In: StatPearls [Internet]. Treasure Island (FL): StatPearls Publishing, 2021. https://www.ncbi.nlm.nih.gov/books/ NBK564350/.

3. Guneli E, Cavdar Z, Islekel H, Sarioglu S, Erbayraktar S, Kiray M, Sokmen S, Yilmaz O and Gokmen N: Erythropoietin protects the intestine against ischemia/reperfusion injury in rats. Mol Med 13: 509-517, 2007.

4. Tahir M, Arshid S, Fontes B, Castro MS, Luz IS, Botelho KLR, Sidoli S, Schwämmle V, Roepstorff P and Fontes W: Analysis of the effect of intestinal ischemia and reperfusion on the rat neutrophils proteome. Front Mol Biosci 5: 89, 2018.

5. Lenaerts K, Ceulemans LJ, Hundscheid IH, Grootjans J, Dejong $\mathrm{CH}$ and Olde Damink SW: New insights in intestinal ischemia-reperfusion injury: Implications for intestinal transplantation. Curr Opin Organ Transplant 18: 298-303, 2013.

6. Liu DM, Sun BW, Sun ZW, Jin Q, Sun Y and Chen X: Suppression of inflammatory cytokine production and oxidative stress by CO-releasing molecules-liberated $\mathrm{CO}$ in the small intestine of thermally-injured mice. Acta Pharmacol Sin 29: 838-846, 2008.

7. Farmer DG, Ke B, Shen XD, Kaldas FM, Gao F, Watson MJ, Busuttil RW and Kupiec-Weglinski JW: Interleukin-13 protects mouse intestine from ischemia and reperfusion injury through regulation of innate and adaptive immunity. Transplantation 91: 737-743, 2011.

8. Arndt H, Kubes P and Granger DN: Involvement of neutrophils in ischemia-reperfusion injury in the small intestine. Klin Wochenschr 69: 1056-1060, 1991.

9. Ucar BI, Erikci A, Kosemehmetoglu K, Ozkul C, Iskit AB Ucar G and Zeren S: Effects of endothelin receptor blockade and COX inhibition on intestinal $\mathrm{I} / \mathrm{R}$ injury in a rat model: Experimental research. Int J Surg 83: 89-97, 2020.

10. Tóth Š, Jonecová Z, Curgali K, Maretta M, Šoltés J, Švaňa M, Kalpadikis T, Caprnda M, Adamek M, Rodrigo L and Kruzliak P: Quercetin attenuates the ischemia reperfusion induced COX-2 and MPO expression in the small intestine mucosa. Biomed Pharmacother 95: 346-354, 2017.

11. Smith WL: A seven-step plan for becoming a moderately rich and famous biochemist. J Biol Chem 294: 1779-1793, 2019.

12. Singh R, Kumar R and Singh DP: Nitric oxide-releasing nonsteroidal anti-inflammatory drugs: Gastrointestinal-sparing potential drugs. J Med Food 12: 208-218, 2009.

13. Takeuchi $\mathrm{K}$ and Amagase K: Roles of cyclooxygenase, prostaglandin E2 and EP receptors in mucosal protection and ulcer healing in the gastrointestinal tract. Curr Pharm Des 24: 2002-2011, 2018.

14. Zhu L, Xu C, Huo X, Hao H, Wan Q, Chen H, Zhang X, Breyer RM, Huang Y,CaoX,etal:Thecyclooxygenase-1/mPGES-1/endothelial prostaglandin EP4 receptor pathway constrains myocardial ischemia-reperfusion injury. Nat Commun 10: 1888, 2019.

15. Chen Y, Wang JC, Yang CM, Fan Q, Zheng J and Liu H: Positive acceleration adaptive training attenuates gastric ischemia-reperfusion injury through COX-2 and PGE2 expression. Exp Ther Med 17: 2901-2906, 2019.

16. Hamada T, Tsuchihashi S, Avanesyan A, Duarte S, Moore C, Busuttil RW and Coito AJ: Cyclooxygenase-2 deficiency enhances Th2 immune responses and impairs neutrophil recruitment in hepatic ischemia/reperfusion injury. J Immunol 180: 1843-1853, 2008

17. Zhang T, Ma Y, Xu KQ and Huang WQ: Pretreatment of parecoxib attenuates hepatic ischemia/reperfusion injury in rats. BMC Anesthesiol 15: 165, 2015.

18. Liu S,Dai Y,Zhou CandZhu T: Parecoxib exhibits anti-inflammatory and neuroprotective effects in a rat model of transient global cerebral ischemia. J Toxicol Environ Health A 83: 203-214, 2020. 
19. Patel NS, Cuzzocrea S, Collino M, Chaterjee PK, Mazzon E, Britti D, Yaqoob MM and Thiemermann C: The role of cycloxygenase-2 in the rodent kidney following ischaemia/reperfusion injury in vivo. Eur J Pharmacol 562: $148-154,2007$

20. Kamel MY, Ahmed SM and Abdelzaher WY: The potential protective effect of modafinil in intestinal ischemic reperfusion-induced in rats. Int Immunopharmacol 88: 106983, 2020.

21. Boivin GP, Hickman DL, Creamer-Hente MA, Pritchett-Corning KR and Bratcher NA: Review of $\mathrm{CO} 2$ as a euthanasia agent for laboratory rats and mice. J Am Assoc Lab Anim Sci 56: 491-499, 2017.

22. Tong F, Dong B, Chai R, Tong K, Wang Y, Chen S, Zhou X and Liu D: Simvastatin nanoparticles attenuated intestinal ischemia/reperfusion injury by downregulating BMP4/COX-2 pathway in rats. Int J Nanomedicine 12: 2477-2488, 2017.

23. Kamel M, Ahmed SM and Abdelzaher W: The potential protective effect of modafinil in intestinal ischemic reperfusion-induced in rats. Int Immunopharmacol 88: 106983, 2020

24. Duarte S, Kato H, Kuriyama N, Suko K, Ishikawa TO Busuttil RW, Herschman HR and Coito AJ: Hepatic ischemia and reperfusion injury in the absence of myeloid cell-derived COX-2 in mice. PLoS One 9: e96913, 2014.

25. Feng Y, Ju H, Yang B and An H: Effects of a selective cyclooxygenase-2 inhibitor on postoperative inflammatory reaction and pain after total knee replacement. J Pain 9: 45-52, 2008.

26. Öztürk T, Vural K, Tuğlu İ, Var A, Kurdal T and Aydemir I: Acute and chronic pretreatment with atenolol attenuates intestinal ischemia and reperfusion injury in hypercholesterolemic rats. J Cardiothorac Vasc Anesth 30: 985-992, 2016.

27. Habes QLM, Linssen V, Nooijen S, Kiers D, Gerretsen J, Pickkers P, Scheffer GJ and Kox M: Markers of intestinal damage and their relation to cytokine levels in cardiac surgery patients. Shock 47: 709-714, 2017.

28. Li Q, Ye T, Long T and Peng X: Ginkgetin exerts anti-inflammatory effects on cerebral ischemia/reperfusion-induced injury in a rat model via the TLR4/NF-kB signaling pathway. Biosci Biotechnol Biochem 83: 675-683, 2019.

29. Vollmar B and Menger MD: Intestinal ischemia/reperfusion: Microcirculatory pathology and functional consequences. Langenbecks Arch Surg 396: 13-29, 2011.

30. Stichtenoth DO: The second generation of COX-2 inhibitors: Clinical pharmacological point of view. Mini Rev Med Chem 4 617-624, 2004.
31. Tyvold SS, Solligård E, Gunnes S, Lyng O, Johannisson A, Grønbech JE and Aadahl P: Bronchial microdialysis of cytokines in the epithelial lining fluid in experimental intestinal ischemia and reperfusion before onset of manifest lung injury. Shock 34: 517-524, 2010.

32. Wang GJ, Deng HY, Maier CM, Sun GH and Yenari MA: Mild hypothermia reduces ICAM-1 expression, neutrophil infiltration and microglia/monocyte accumulation following experimental stroke. Neuroscience 114: 1081-1090, 2002.

33. Sun Y, Xu Q, Wu Z, Gong Y and Tang L: Parecoxib inhibits inflammatory responses in a mouse model of sepsis. FEBS Open Bio: Apr 3, 2020 (Epub ahead of print).

34. Kimura M, Yokoyama A and Higuchi S: Aldehyde dehydrogenase-2 as a therapeutic target. Expert Opin Ther Targets 23: 955-966, 2019.

35. Wallace JL, Ianaro A and de Nucci G: Gaseous mediators in gastrointestinal mucosal defense and injury. Dig Dis Sci 62: 2223-2230, 2017.

36. Lau A, Arundine M, Sun HS, Jones M and Tymianski M: Inhibition of caspase-mediated apoptosis by peroxynitrite in traumatic brain injury. J Neurosci 26: 11540-11553, 2006.

37. Liu W, Fan Z, Han Y, Lu S, Zhang D, Bai X, Xu W, Li J and Wang H: Curcumin attenuates peroxynitrite-induced neurotoxicity in spiral ganglion neurons. Neurotoxicology 32: 150-157, 2011.

38. Borisov VB, Siletsky SA, Nastasi MR and Forte E: ROS defense systems and terminal oxidases in bacteria. Antioxidants (Basel) 10: 839, 2021.

39. Pérez S, Taléns-Visconti R, Rius-Pérez S, Finamor I and Sastre J: Redox signaling in the gastrointestinal tract. Free Radic Biol Med 104: 75-103, 2017.

40. Wu F, Wang W, Duan Y, Guo J, Li G and Ma T: Effect of parecoxib sodium on myocardial ischemia-reperfusion injury rats. Med Sci Monit 27: e928205, 2021.

41. Luo CC, Huang CS, Ming YC, Chu SM and Chao HC: Calcitonin gene-related peptide downregulates expression of inducible nitride oxide synthase and caspase-3 after intestinal ischemia-reperfusion injury in rats. Pediatr Neonatol 57: 474-479, 2016.

This work is licensed under a Creative Commons Attribution-NonCommercial-NoDerivatives 4.0 International (CC BY-NC-ND 4.0) License. 